\title{
AVALIAÇÃO DA APLICAÇÃO DE FUNGICIDA ÀS SEMENTES DE AMENDOIM ANTES DO ENVELHECIMENTO ACELERADO ${ }^{1}$
}

\author{
CLAUDIA A. V. ROSSETTO², ANTONIO E.S. ARAÚJO³ ${ }^{3}$ TATIANA M. LIMA
}

\begin{abstract}
RESUMO - Os objetivos do trabalho foram os de avaliar o efeito da aplicação de fungicida às sementes de amendoim (Arachis hypogaea L.), que foram colhidas em distintos estádios de maturação e provenientes de plantas-mãe que foram ou não submetidas à calagem, nas condições de envelhecimento acelerado. Foram avaliados quatro lotes de sementes do cultivar Botucatu, provenientes das áreas que receberam (CC) ou não a aplicação de calcário (SC) e que foram colhidas aos 104 (E1) e 124 (E2) dias após a semeadura e denominados de lote 1 (E1/SC), 2 (E1/ CC), 3 (E2/SC) e 4(E2/CC). De cada lote, foram retiradas duas amostras de sementes, uma original e outra tratada com fungicida thiram. Estas amostras foram submetidas às condições do teste de envelhecimento acelerado, e após o período de exposição, as sementes foram avaliadas na primeira e na segunda contagem do teste de germinação realizado com e sem secagem. Pelos resultados pode-se concluir que há maior incidência de patógenos nas sementes colhidas aos 104 DAS e provenientes de plantas-mãe submetidas à calagem; o tratamento fungicida favorece a germinação das sementes contaminadas por Aspergillus do grupo flavus e Aspergillus niger, até 24 horas após a exposição às condições de envelhecimento acelerado.

Termos para indexação: Arachis hypogaea, germinação, sanidade, vigor.
\end{abstract}

\section{EVALUATION OF THE FUNGICIDE APPLICATION TO PEANUT SEEDS BEFORE ACCELERATED AGING}

\begin{abstract}
The objective of this research was to evaluate the effects of fungicide application on peanut seeds (Arachis hypogaea L.) that were harvested at different maturity stages and obtained from plants submitted to lime, in the conditions of accelerated aging. For this, four peanut seeds lots of cv. Botutatu were evaluated, obtained from areas that received (CC) or not lime application (SC) and that were harvested at 104 (E1) and 124 (E2) days after sowing and called lot 1 (E1/SC), 2 (E1/CC), 3 (E2/SC) and 4 (E2/CC). Each lot was divided into two seeds samples, an original and another that was dressed with thiram. Samples were submitted to accelerated aging conditions and evaluated just after and the after drying. The results allowed the conclusion that there is greatest fungal incidence in the seeds that were harvested at 104 days after sowing and derived from plants that were submitted to lime. The fungicide application favored the germination of seeds that were contaminated by Aspergillus of the flavus group and for Aspergillus niger until 24 hours after exposure to accelerated aging conditions.
\end{abstract}

Index terms: Arachis hypogaea; germination; sanity; vigor.

\section{INTRODUÇÃO}

${ }^{1}$ Aceito para publicação em 08.07.2003.

${ }^{2}$ Eng $^{0}$ Agr $^{\circ}$, Dr., Pesquisador do IAPAR; Cx. Postal 129, 84001-970, Ponta Grossa, PR; e-mail: dahrens@pr.gov.br

${ }^{3}$ Eng ${ }^{\circ}$ Agrícola, Dr., Prof. Adjunto, Depto. de Fitotecnia, FAEM/UFPel, Cx. Postal 354, 96010-900, Pelotas, RS.

${ }^{4}$ Eng $^{\circ}$ Agr $^{\circ}$, Dr., Prof. Adjunto, Depto. de Fitotecnia e Fitossanitarismo, SSA/UFPR, Rua dos Funcinários 1540, 80035-050, Curitiba, PR.
A produção de sementes de amendoim demanda alta tecnologia para evitar vários riscos que usualmente ocorrem desde a colheita até a comercialização. Como a maioria das leguminosas, o amendoim mostra uma rápida perda da viabilidade durante o armazenamento, dependendo do conteúdo de água, das condições ambientais e da aplicação de fungicida (Usberti \& Amaral, 1999). 
O teste de envelhecimento acelerado tem sido considerado eficiente para avaliar o vigor de lotes de sementes, pois ao mesmo tempo em que identifica pequenas diferenças de vigor, pode estimar o potencial de conservação das sementes. No entanto, dentro e entre laboratório, tem-se verificado que este teste apresenta resultados discordantes devido a causas, tais como, a cultivar utilizada (Mello \& Tilmann, 1987), o momento de encerramento da análise, se na primeira ou na segunda contagem do teste de germinação (Marcos-Filho et al., 1999), o tempo de permanência das sementes após estas serem retiradas da câmara de envelhecimento (Scott, 1981; Nascimento-Junior et al., 1998; Rossetto et al., 2001), a origem das sementes (Usberti \& Amaral, 1999) e a qualidade fisiológica das sementes (Tekrony et al., 1984 e Aguero et al., 1997), que é influenciada, dentre outros, pelas condições ambientais prevalecentes durante a fase de maturação e pela nutrição da planta-mãe (Carvalho \& Nakagawa, 1999).

Além disso, a condução do teste de envelhecimento acelerado, sob condições de elevadas temperatura e umidade relativa do ar, ocasiona a deterioração das sementes e favorece o aparecimento, na germinação subseqüente, de anormalidade ou morte (Gupta et al., 1993). Para Marcos-Filho (1994), as condições impostas por esse teste não agem apenas no comportamento das sementes, mas também influenciam na ação de microrganismos participantes da deterioração, uma vez que temperatura e umidade elevadas podem interferir na manifestação de alguns microrganismos associados às sementes. Silva \& Silva (2000) e Rossetto et al. (2001) constataram que a alta incidência de Aspergillus spp. e de Rhizopus spp. pode prejudicar a avaliação do teste de envelhecimento acelerado em sementes de feijão e de amendoim, respectivamente. No entanto, tem sido verificado menor contaminação inicial em lotes de sementes de amendoim, que foram provenientes de plantas-mãe que receberam a aplicação de calcário, visando principalmente o fornecimento de cálcio (Fernandes et al., 1997). Pitt et al. (1991) constataram que o maior conteúdo deste macronutriente nas sementes pode diminuir o crescimento de fungos, principalmente de Aspergillus spp. Além disso, na presença de cálcio, Small et al. (1989) observaram que o pericarpo do fruto torna-se mais espesso.

Considerando a aplicação de fungicida às sementes antes destas serem colocadas na câmara de envelhecimento, temse que este tratamento químico tende a favorecer a germinação das sementes infectadas com Aspergillus glaucus e com Aspergillus niger, que foram submetidas ao envelhecimento acelerado (Marcos Filho \& Shioga (1981); Gupta et al., 1993).

Com base no exposto, o objetivo do trabalho foi o de avaliar o efeito da aplicação de fungicida às sementes de amendoim, que foram colhidas em distintos estádios de maturação e provenientes de plantas-mãe que foram ou não submetidas à calagem, nas condições de envelhecimento acelerado.

\section{MATERIAL E MÉTODOS}

O trabalho foi conduzido em condições de laboratório, avaliando-se quatro lotes de sementes de amendoim (Arachis hypogaea L.), cv. Botutatu (Zanotto, 1993), provenientes das áreas que receberam (CC) ou não a aplicação de calcário (SC) e que foram colhidas aos 104 (E1) e 124 (E2) dias após a semeadura, respectivamente em 13/7/01 e 03/08/01 e, denominados de lote 1 (E1/SC), 2 (E1/CC), 3 (E2/SC) e 4(E2/CC). De cada lote, foram retiradas duas amostras de sementes, uma original e outra que foi tratada com fungicida thiram (Rhodiauram 70\%), na forma de pó-seco, à dose de $210 \mathrm{~g}$ do i.a por $100 \mathrm{~kg}$ de sementes. Em seguida, as sementes foram submetidas aos testes de grau de umidade, de germinação e de sanidade, com base em Brasil (1992) e aos testes de vigor (primeira contagem de germinação e condutividade elétrica), com base em Krzyzanowski et al. (1991) e em Vanzolini \& Nakagawa (1998), respectivamente.

O teor de água foi determinado com duas repetições de sementes, pelo método da estufa a $105 \pm 3^{\circ} \mathrm{C}$, por 24 horas (Brasil, 1992). $O$ teste de germinação foi realizado com 25 sementes por repetição, sob temperatura de $25^{\circ} \mathrm{C}$, utilizando substrato de papel tipo germitest em forma de rolo, umedecido na proporção de três vezes o peso do papel em volume de água, conforme os procedimentos descritos em Brasil (1992). O teste de primeira contagem foi conduzido conjuntamente com o teste de germinação, considerando a porcentagem de plântulas normais na primeira contagem estabelecida em Brasil (1992). O teste de condutividade elétrica foi conduzido com a utilização de 25 sementes por repetição. Após a pesagem, as sementes foram imersas em 75 mL de água destilada para permanência em germinador, durante 24 horas, à temperatura de $20^{\circ} \mathrm{C}$. Ao término do período, a condutividade elétrica da solução foi determinada em condutivímetro; os valores obtidos foram expressos em uS/cm/g de sementes (Vanzolini \& Nakagawa, 1998). O teste de sanidade foi realizado com base em metodologia descrita em Ito et al (1992). Por este método a sementes são incubadas a $25^{\circ} \mathrm{C}$, por 5 dias sob regime luminoso de 12 horas. Por repetição, foram utilizadas 10 sementes, colocadas em caixas gerbox, contendo papel de filtro umedecido com água destilada. A avaliação foi feita sob microscópios, observando-se as estruturas dos patógenos. 
Para a condução do teste de envelhecimento acelerado, as sementes foram dispostas em camada única sobre a tela do interior de mini-câmaras (gerbox adaptado, com 40 $\mathrm{ml}$ de água destilada em seu fundo) e mantidas em B.O.D., a $42^{\circ} \mathrm{C}$, por 72 horas. Após os períodos de exposição, as sementes permaneceram sobre a tela do gerbox, em condições de ambiente de laboratório $\left(25^{\circ} \mathrm{C}\right.$ e $\left.78 \%\right)$, por zero, 24 e 48 horas, para secagem. Posteriormente, com base em Brasil (1992), foram instalados os testes de grau de umidade e de germinação, visando calcular, respectivamente, o teor de água após a saída das sementes da câmara de envelhecimento e a porcentagem de plântulas normais no quinto e, também, no décimo dia. Conjuntamente, foi realizado o teste de sanidade, que consistiu na identificação de patógenos, avaliando-se as estruturas nas sementes e nas plântulas, sob microscópios (Brasil, 1992). Considerou-se a incidência de Aspergillus niger, Aspergillus do grupo flavus, Penicillium spp. e Rhizopus spp. (Sing et al., 1992).

O delineamento experimental adotado foi inteiramente ao acaso, em esquema fatorial (4 lotes x 2 amostras), com quatro repetições. A análise de variância foi conduzida isoladamente em cada período de secagem. Para a comparação de médias dos tratamentos dos vários parâmetros estudados, foi adotado o teste Tukey, a 5 \% de probabilidade.

\section{RESULTADO E DISCUSSÃO}

Quando foi realizada a avaliação antes do teste de envelhecimento acelerado, não houve interação entre lotes de sementes de amendoim e amostras destes lotes (original e tratada com fungicida) no teor de água e na germinação e no vi- gor (Tabela 1). Independente da amostra, foi constatado menor vigor das sementes dos lotes 1 e 2 , avaliado pela menor porcentagem de plântulas normais na primeira contagem e pelo maior valor de condutividade elétrica na solução de embebição destas sementes. As sementes destes lotes foram colhidas aos 104 dias após a semeadura (DAS). Segundo Rossetto et al. (1994), a máxima qualidade fisiológica das sementes de amendoim foi observada aos 129 DAS. Além disso, nas colheitas destas sementes, foi constatado que ocorreu 7,0 mm de precipitação pluvial e que a temperatura média foi de $18^{\circ} \mathrm{C}$. Já, nas colheitas dos lotes 3 e 4 não ocorreu precipitação pluvial e a temperatura média foi de $24^{\circ} \mathrm{C}$ (dados não apresentados). Em soja, Tekrony et al. (1984) e Aguero et al. (1997) constataram que as diferenças na qualidade fisiológica entre lotes de sementes podem ser atribuídas principalmente aos efeitos das condições ambientais prevalecentes durante a fase de maturação e colheita.

Também pela Tabela 1, pode-se verificar que não houve efeitos significativos dos tratamentos no grau de umidade das sementes e que, independente da amostra, foi constatado menor germinação das sementes do lote 1, provavelmente, em função da maior porcentagem de plântulas anormais (total e infeccionada). Além disso, independente do lote, a maior porcentagem de germinação e a menor de plântulas anormais (total e infeccionada) foram provenientes de sementes tratadas com fungicida.

Em relação à contaminação inicial, pode-se constatar que houve interação entre lotes e amostras na porcentagem de Aspergillus spp. nas sementes (Tabela 2). As sementes dos lotes 1 e 3, que não foram tratadas (original), apresentaram maiores incidências de Aspergillus niger e de Aspergillus do

TABELA 1. Dados médios de grau de umidade (\%), de plântulas normais na primeira contagem (\%), de germinação (\%), de plântulas anormais (total - P.A.T e infeccionadas - P.A.I) (\%) e de condutividade elétrica (C.E. $\mu \mathrm{S} / \mathrm{cm} / \mathrm{g})$, obtidos antes do envelhecimento das sementes de amendoim de duas amostras, uma original (SF) e outra que foi tratada com fungicida (CF), provenientes de quatro diferentes lotes (L1, L2, L3 e L). Seropédica. 2001.

\begin{tabular}{lcccccc}
\hline Tratamentos & Grau de umidade & Primeira contagem & Germinação & P.A.T & P.A.I. & C.E. \\
\hline Lotes & & & & & & \\
L1(E1/SC) & $7.2 \mathrm{a}$ & $15 \mathrm{~b}$ & $59 \mathrm{~b}$ & $41 \mathrm{a}$ & $36 \mathrm{a}$ & $25.26 \mathrm{a}$ \\
L2(E1/CC) & $7.4 \mathrm{a}$ & $16 \mathrm{~b}$ & $84 \mathrm{a}$ & $16 \mathrm{~b}$ & $13 \mathrm{~b}$ & $22.33 \mathrm{a}$ \\
L3(E2/SC) & $7.3 \mathrm{a}$ & $27 \mathrm{a}$ & $80 \mathrm{a}$ & $20 \mathrm{~b}$ & $19 \mathrm{~b}$ & $21.48 \mathrm{~b}$ \\
L4(E2/CC) & $7.0 \mathrm{a}$ & $25 \mathrm{a}$ & $90 \mathrm{a}$ & $13 \mathrm{~b}$ & $10 \mathrm{~b}$ & $21.40 \mathrm{~b}$ \\
Amostras & & & & & & \\
SF & $7.3 \mathrm{~A}$ & $21 \mathrm{~A}$ & $70 \mathrm{~B}$ & $30 \mathrm{~A}$ & $26 \mathrm{~A}$ & $22.90 \mathrm{~A}$ \\
CF & $7.1 \mathrm{~A}$ & $21 \mathrm{~A}$ & $86 \mathrm{~A}$ & 16 B & 14 B & $23.34 \mathrm{~A}$ \\
\hline CV (\%) & 6.48 & 2.75 & 3.01 & 3.92 & 4.18 & 9.91 \\
\hline
\end{tabular}

Médias seguidas pela mesma letra (minúsculas para lotes e maiúsculas para amostras) não diferem entre si pelo teste Tukey, a 5\%. 
TABELA 2. Dados médios de porcentagem de fungos obtidos antes do envelhecimento das sementes de amendoim de duas amostras, uma original (SF) e outra que foi tratada com fungicida (CF), provenientes de quatro diferentes lotes (L1, L2, L3 e L4). Seropédica. 2001.

\begin{tabular}{|c|c|c|c|c|c|c|c|}
\hline \multirow{2}{*}{ Tratamentos } & \multirow{2}{*}{ Penicillium spp. } & \multirow{2}{*}{ Fusarium spp. } & \multirow{2}{*}{ Rhizopus spp. } & \multicolumn{2}{|c|}{ Aspergillus niger } & \multicolumn{2}{|c|}{ Aspergillus do grupo flavus } \\
\hline & & & & SF & CF & $\mathrm{SF}$ & $\mathrm{CF}$ \\
\hline \multicolumn{8}{|l|}{ Lotes } \\
\hline L1(E1/SC) & $7 \mathrm{a}$ & $14 \mathrm{a}$ & $19 \mathrm{a}$ & $24 \mathrm{Aa}$ & $0 \mathrm{Ba}$ & $11 \mathrm{Aa}$ & $0 \mathrm{Ba}$ \\
\hline L2(E1/CC) & $1 b$ & $2 b$ & $10 \mathrm{~b}$ & $0 \mathrm{Ab}$ & $0 \mathrm{Aa}$ & $0 \mathrm{Ab}$ & $0 \mathrm{Aa}$ \\
\hline L3(E2/SC) & $2 b$ & $1 \mathrm{~b}$ & $5 b$ & $6 \mathrm{Ab}$ & $1 \mathrm{Ba}$ & $4 \mathrm{Ab}$ & $0 \mathrm{Ba}$ \\
\hline L4(E2/CC) & $0 \mathrm{~b}$ & $0 \mathrm{~b}$ & $10 \mathrm{~b}$ & $0 \mathrm{Ab}$ & $0 \mathrm{Aa}$ & $0 \mathrm{Ab}$ & $0 \mathrm{Aa}$ \\
\hline \multicolumn{8}{|l|}{ Amostras } \\
\hline SF & $5 \mathrm{~A}$ & $4 \mathrm{~A}$ & $12 \mathrm{~A}$ & & & & \\
\hline CF & 0B & $4 \mathrm{~A}$ & $10 \mathrm{~A}$ & & & & \\
\hline CV (\%) & 1.46 & 1.62 & 2.61 & \multicolumn{2}{|c|}{0.99} & \multicolumn{2}{|c|}{0.86} \\
\hline
\end{tabular}

Médias seguidas pela mesma letra (minúsculas para lotes e maiúsculas para amostras) não diferem entre si pelo teste Tukey, a 5\%.

grupo flavus do que as que foram tratadas com fungicida. As sementes destes dois lotes foram obtidas de plantas-mãe que não receberam a aplicação de calcário, visando principalmente o fornecimento de cálcio. Para Pitt et al. (1991), o maior conteúdo de cálcio nas sementes pode diminuir o crescimento de fungos, principalmente de Aspergillus spp. Além disso, na presença de cálcio, Small et al. (1989) observaram que o pericarpo do fruto torna-se mais espesso.

Também, pode-se constatar na Tabela 2 que independente da amostra, maior incidência de Penicillium spp., de Fusarium spp. e de Rhizopus spp. nas sementes do lote 1, podendo esta contaminação inicial determinar a perda de viabilidade dessas sementes, como constatado na Tabela 1.

Quando a análise do envelhecimento acelerado foi encerrada na primeira contagem do teste de germinação conduzido após zero e 48 horas de secagem, foi possível identificar interação entre lotes e amostras (Tabela 3). As maiores porcentagens de plântulas normais foram provenientes das sementes dos quatro lotes que foram tratadas do que das sementes das amostras originais. No entanto, após 24 horas de secagem, não foi possível identificar efeitos significativos dos tratamentos isolados (Tabela 3).

Quando a análise foi encerrada na segunda contagem do teste de germinação, conduzido por zero e 24 horas de secagem, foi constatado que houve interação entre lotes e o tratamento fungicida (Tabela 4). As maiores porcentagens de plântulas normais foram provenientes das sementes dos lotes 1 e 3 , que foram tratadas com fungicida do que das sementes das amostras originais, assim como observado para as sementes destes dois lotes, quando a análise foi encerrada na pri- meira contagem do teste realizado após zero e 48 horas de secagem (Tabela 3). Para os demais lotes não ocorreu diferença significativa entre a amostra tratada e a não tratada. Em sementes de soja, Gupta et al. (1993) constataram que o tratamento químico, empregando os fungicidas benomil, thiram e captan, favoreceu a germinação das sementes infectadas com Aspergillus glaucus e com Aspergillus niger, que foram submetidas ao envelhecimento acelerado.

TABELA 3. Dados médios de porcentagem de plântulas normais por ocasião do encerramento da análise na primeira contagem do teste de germinação, realizado após zero, 24 e 48 horas de secagem, empregando as sementes de amendoim de duas amostras, uma original (SF) e outra que foi tratada com fungicida (CF), provenientes de quatro diferentes lotes (L1, L2, L3 e L4). Seropédica. 2001.

\begin{tabular}{|c|c|c|c|c|c|}
\hline \multirow{2}{*}{ Tratamentos } & \multicolumn{2}{|c|}{ Oh } & \multirow{2}{*}{$24 \mathrm{~h}$} & \multicolumn{2}{|c|}{$48 \mathrm{~h}$} \\
\hline & SF & $\mathrm{CF}$ & & SF & $\mathrm{CF}$ \\
\hline \multicolumn{6}{|l|}{ Lotes } \\
\hline L1(E1/SC) & $9 \mathrm{Bb}$ & $26 \mathrm{Aa}$ & $18 \mathrm{a}$ & $9 \mathrm{Bb}$ & $16 \mathrm{Aa}$ \\
\hline L2(E1/CC) & $12 \mathrm{Ba}$ & $24 \mathrm{Aa}$ & $16 \mathrm{a}$ & $10 \mathrm{Ba}$ & $20 \mathrm{Aa}$ \\
\hline L3(E2/SC) & $18 \mathrm{Ba}$ & $32 \mathrm{Aa}$ & $28 a$ & $14 \mathrm{Ba}$ & $22 \mathrm{Aa}$ \\
\hline L4(E2/CC) & $18 \mathrm{Ba}$ & $32 \mathrm{Aa}$ & $21 \mathrm{a}$ & $16 \mathrm{Ba}$ & $24 \mathrm{Aa}$ \\
\hline \multicolumn{6}{|l|}{ Amostras } \\
\hline $\mathrm{SF}$ & & & $18 \mathrm{~A}$ & & \\
\hline CF & & & $19 \mathrm{~A}$ & & \\
\hline CV (\%) & \multicolumn{2}{|c|}{6.60} & 3.60 & \multicolumn{2}{|c|}{5.52} \\
\hline
\end{tabular}

Médias seguidas pela mesma letra (minúsculas para lotes e maiúsculas para amostras) não diferem entre si pelo teste Tukey, a 5\%. 
TABELA 4. Dados médios de porcentagem de plântulas normais por ocasião do encerramento da análise na segunda contagem do teste de germinação, realizado após zero, 24 e 48 horas de secagem, empregando as sementes de amendoim de duas amostras, uma original (SF) e outra que foi tratada com fungicida (CF), provenientes de quatro diferentes lotes (L1, L2, L3 e L4). Seropédica. 2001.

\begin{tabular}{|c|c|c|c|c|c|}
\hline \multirow{2}{*}{ Tratamentos } & \multicolumn{2}{|c|}{ Oh } & \multicolumn{2}{|c|}{$24 \mathrm{~h}$} & \multirow{2}{*}{$48 \mathrm{~h}$} \\
\hline & SF & CF & SF & CF & \\
\hline \multicolumn{6}{|l|}{ Lotes } \\
\hline L1(E1/SC) & $48 \mathrm{Bb}$ & $67 \mathrm{Aa}$ & $38 \mathrm{Bb}$ & 63Аа & $48 b$ \\
\hline L2(E1/CC) & $80 \mathrm{Aa}$ & 86Aa & 73Aa & 83Аа & $79 a$ \\
\hline L3(E2/SC) & $66 \mathrm{Ba}$ & 84Aa & $45 \mathrm{Ba}$ & 77Aa & $70 a$ \\
\hline L4(E2/CC) & $87 \mathrm{Aa}$ & $91 \mathrm{Aa}$ & 79Аа & 89Аа & $80 a$ \\
\hline \multicolumn{6}{|l|}{ Amostras } \\
\hline SF & & & & & $58 \mathrm{~B}$ \\
\hline CF & & & & & $80 \mathrm{~A}$ \\
\hline CV (\%) & \multicolumn{2}{|c|}{2.55} & \multicolumn{2}{|c|}{3.71} & 3.10 \\
\hline
\end{tabular}

Médias seguidas pela mesma letra (minúsculas para lotes e maiúsculas para amostras) não diferem entre si pelo teste Tukey, a 5\%.

Em relação à condução do teste de germinação após 48 horas de secagem, foi constatada, independente da amostra, menor porcentagem de plântulas normais provenientes das sementes do lote 1 , que apresentaram-se com menor vigor (Tabela 1) e com maior incidência de fungos (Tabela 2). No entanto, Spinola et al. (2000) não constataram incidência de patógenos que pudesse interferir no desempenho das sementes de milho, provavelmente devido ao tratamento fungicida e a desinfecção prévia com hipoclorito de sódio, a 1\%, que as sementes receberam antes do envelhecimento acelerado.

Pela Tabela 5, constata-se que não houve efeito dos tratamentos para o grau de umidade das sementes após a saída destas da câmara de envelhecimento. Assim, provavelmente, não ocorreram diferenças na intensidade de deterioração durante o envelhecimento, como constatado por Marcos-Filho (1999), que as sementes mais úmidas, em geral, são mais sensíveis às condições do teste. Após 48 horas de permanência em condições de laboratório, o teor de água das sementes situava-se na faixa de 18,5 a 18,9\%, que pode justificar a interrupção ao processo de
TABELA 5. Dados médios de grau de umidade -\% após zero, 24 e 48 horas de secagem das sementes de amendoim de duas amostras, uma original (SF) e outra que foi tratada com fungicida (CF), provenientes de quatro diferentes lotes (L1, L2, L3 e L4). Seropédica. 2001.

\begin{tabular}{lccc}
\hline Tratamentos & $0 \mathrm{~h}$ & $24 \mathrm{~h}$ & $48 \mathrm{~h}$ \\
\hline Lotes & & & \\
L1(E1/SC) & $20.6 \mathrm{a}$ & $19.9 \mathrm{a}$ & $18.8 \mathrm{a}$ \\
L2(E1/CC) & $20.3 \mathrm{a}$ & $19.9 \mathrm{a}$ & $18.5 \mathrm{a}$ \\
L3(E2/SC) & $20.4 \mathrm{a}$ & $19.8 \mathrm{a}$ & $18.7 \mathrm{a}$ \\
L4(E2/CC) & $20.4 \mathrm{a}$ & $19.9 \mathrm{a}$ & $18.9 \mathrm{a}$ \\
Amostras & & & \\
SF & $20.3 \mathrm{~A}$ & $19.9 \mathrm{~A}$ & $18.9 \mathrm{~A}$ \\
CF & $20.5 \mathrm{~A}$ & $19.9 \mathrm{~A}$ & $18.6 \mathrm{~A}$ \\
\hline CV $(\%)$ & 7.02 & 3.53 & 4.52 \\
\hline
\end{tabular}

Médias seguidas pela mesma letra (minúsculas para lotes e maiúsculas para amostras) não diferem entre si pelo teste Tukey, a 5\%.

deterioração após a saída das sementes da câmara de envelhecimento.

A análise da freqüência dos fungos das plântulas deterioradas e das sementes mortas (Tabela 6) revela que houve interação entre lotes e amostras para Aspergillus spp. As se-

TABELA 6. Dados médios de porcentagem de fungos encontrados nas sementes e nas plântulas de amendoim de duas amostras, uma original (SF) e outra que foi tratada com fungicida (CF), provenientes de quatro diferentes lotes (L1, L2, L3 e L4). Avaliações realizadas por ocasião do encerramento da análise na $2^{\mathrm{a}}$ contagem do teste de germinação realizado após zero, 24 e 48 horas de secagem. Seropédica. 2001.

\begin{tabular}{|c|c|c|c|c|c|c|}
\hline \multirow{2}{*}{ Lotes } & \multicolumn{2}{|c|}{ Oh } & \multicolumn{2}{|c|}{$24 \mathrm{~h}$} & \multicolumn{2}{|c|}{$48 \mathrm{~h}$} \\
\hline & SF & $\mathrm{CF}$ & SF & $\mathrm{CF}$ & SF & CF \\
\hline & \multicolumn{6}{|c|}{ Aspergillus niger .. } \\
\hline L1(E1/SC) & $15 \mathrm{Aa}$ & $0 \mathrm{Ba}$ & $18 \mathrm{Aa}$ & $0 \mathrm{Ba}$ & $16 \mathrm{Aa}$ & $0 \mathrm{Ba}$ \\
\hline L2(E1/CC) & $1 \mathrm{Ab}$ & $0 \mathrm{Aa}$ & $6 \mathrm{Ab}$ & $0 \mathrm{Aa}$ & $2 \mathrm{Ab}$ & $0 \mathrm{Aa}$ \\
\hline L3(E2/SC) & $7 \mathrm{Ab}$ & $0 \mathrm{Aa}$ & $10 \mathrm{Ab}$ & $0 \mathrm{Aa}$ & $12 \mathrm{Aab}$ & $0 \mathrm{Ba}$ \\
\hline L4(E2/CC) & $3 \mathrm{Ab}$ & $0 \mathrm{Aa}$ & $6 \mathrm{Ab}$ & $0 \mathrm{Aa}$ & $1 \mathrm{Ab}$ & $0 \mathrm{Aa}$ \\
\hline \multirow[t]{2}{*}{ CV (\%) } & \multicolumn{2}{|c|}{1.02} & \multicolumn{2}{|c|}{2.13} & \multicolumn{2}{|c|}{1.61} \\
\hline & \multicolumn{6}{|c|}{...... Aspergillus do grupo flavus .......... } \\
\hline L1(E1/SC) & $13 \mathrm{Aa}$ & $0 \mathrm{Ba}$ & $15 \mathrm{Aa}$ & $0 \mathrm{Ba}$ & $14 \mathrm{Aa}$ & $0 \mathrm{Ba}$ \\
\hline L2(E1/CC) & $1 \mathrm{Ab}$ & $0 \mathrm{Aa}$ & $2 \mathrm{Ab}$ & $0 \mathrm{Aa}$ & $2 \mathrm{Ab}$ & $0 \mathrm{Aa}$ \\
\hline L3(E2/SC) & $11 \mathrm{Aa}$ & $0 \mathrm{Ba}$ & $5 \mathrm{Ab}$ & $0 \mathrm{Aa}$ & $18 \mathrm{Aa}$ & $0 \mathrm{Ba}$ \\
\hline L4(E2/CC) & $1 \mathrm{Ab}$ & $0 \mathrm{Aa}$ & $2 \mathrm{Ab}$ & $0 \mathrm{Aa}$ & $2 \mathrm{Aa}$ & $0 \mathrm{Aa}$ \\
\hline CV (\%) & \multicolumn{2}{|c|}{0.65} & \multicolumn{2}{|c|}{1.01} & \multicolumn{2}{|c|}{1.81} \\
\hline
\end{tabular}

Médias seguidas pela mesma letra (minúsculas para lotes e maiúsculas para amostras) não diferem entre si pelo teste Tukey, a 5\%. 
mentes do lote 1 , que não foram tratadas (original), apresentaram maior incidência destes fungos, em relação as sementes tratadas com fungicida, por ocasião da segunda contagem do teste de germinação realizado após zero, 24 e 48 horas. Além disso, não houve efeitos significativos dos tratamentos isolados para Fusarium spp. e Rhizopus spp. (Tabela 7). No entanto, independente do lote, foi constatada maior incidência de Penicillium spp. nas sementes que não foram tratadas com fungicida, assim como constatado por ocasião da avaliação inicial (Tabela 2). De maneira geral, pode-se constatar o aumento da incidência de Aspergillus spp. e a diminuição dos demais fungos, com o envelhecimento. Silva \& Silva (2000) verificaram que as condições de envelhecimento acelerado favorecem o desenvolvimento de microrganismos e, destacam a tendência do domínio do Aspergillus spp. sobre os demais fungos presentes.

\section{CONCLUSÕES}

Há maior incidência de patógenos nas sementes colhidas aos 104 DAS e provenientes de plantas-mãe submetidas à calagem;

O tratamento fungicida favorece a germinação das sementes contaminadas por Aspergillus do grupo flavus e Aspergillus niger, até 24 horas após a exposição às condições de envelhecimento acelerado.

\section{REFERÊNCIAS}

AGUERO, J.A.P.; VIEIRA, R.D.; BITTENCOURT, S.R. Avaliação da qualidade fisiológica de sementes de cultivares de soja. Revista Brasileira de Sementes, Brasília, v.19, n.2, p.255-260, 1997.

BRASIL. Ministério da Agricultura e Reforma Agrária. Regras para análise de sementes. Brasília: SNDA/DNDV/CLAV, 1992. 365p.

CARVALHO, N.M.; NAKAGAWA, J. Sementes: ciência, tecnologia e produção. Jaboticabal: FUNEP, 1999. 326p.

FERNANDEZ, E.; ROSOLEM, C.A.; MARINGONI, A.C.; OLIVEIRA, D.M.T. Fungus incidence on peanut grains as affected by drying method and Ca nutrition. Field Crops Research, Amesterdan, v.52, p.9-15, 1997.

GUPTA, I.J.; SCHMITTHENNER, A.F.; McDONALD, M.B. Effect of storage fungi on seed vigour of soybean. Seed Science and Technology, Zürich, v.21, n.3, p.581-591, 1993.

ITO, M.F.: BACCHI, L. M.A.; MARINGONI, A.C.; MENTEN, J.O.M. Comparação de métodos para detecção de Aspergillus spp. e Penicillium spp. em sementes de amendoim (Arachis hypogaea L.) Summa Phytopathologica, v.18, n.3, p.262-268, 1992.

KRZYZANOWSKI, F.C.; FRANÇA-NETO, J.B.; HENNING, A.A. Relato dos testes de vigor para as grandes culturas. Informativo
TABELA 7. Dados médios de porcentagem de fungos encontrados nas sementes e plântulas de amendoim de duas amostras, uma original (SF) e outra que foi tratada com fungicida (CF), provenientes de quatro diferentes lotes (L1, L2, L3 e L4). Avaliações realizadas por ocasião do encerramento da análise na $2^{a}$ contagem do teste de germinação realizado após zero, 24 e 48 horas de secagem. Seropédica. 2001.

\begin{tabular}{|c|c|c|c|}
\hline Tratamentos & Oh & $24 \mathrm{~h}$ & $48 \mathrm{~h}$ \\
\hline & \multicolumn{3}{|c|}{............... Penicillium spp...................... } \\
\hline \multicolumn{4}{|l|}{ Lotes } \\
\hline L1 (E1/SC) & $2 \mathrm{a}$ & $2 \mathrm{a}$ & $2 \mathrm{a}$ \\
\hline $\mathrm{L} 2(\mathrm{E} 1 / \mathrm{CC})$ & $2 a$ & $2 \mathrm{a}$ & $2 \mathrm{a}$ \\
\hline $\mathrm{L} 3(\mathrm{E} 2 / \mathrm{SC})$ & $2 \mathrm{a}$ & $0 \mathrm{a}$ & $3 a$ \\
\hline $\mathrm{L} 4(\mathrm{E} 2 / \mathrm{CC})$ & $0 \mathrm{a}$ & $0 \mathrm{a}$ & $2 \mathrm{a}$ \\
\hline \multicolumn{4}{|l|}{ Amostras } \\
\hline SF & $4 \mathrm{~A}$ & $2 \mathrm{~A}$ & $5 \mathrm{~A}$ \\
\hline CF & 0B & oB & $0 \mathrm{~B}$ \\
\hline \multirow[t]{2}{*}{ CV (\%) } & 0.66 & 0.99 & 2.04 \\
\hline & \multicolumn{3}{|c|}{ 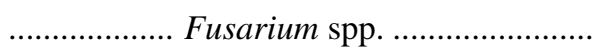 } \\
\hline \multicolumn{4}{|c|}{ (at } \\
\hline L1(E1/SC) & $1 \mathrm{a}$ & $1 \mathrm{a}$ & $0 \mathrm{a}$ \\
\hline L2(E1/CC) & $2 \mathrm{a}$ & $0 \mathrm{a}$ & $1 \mathrm{a}$ \\
\hline L3(E2/SC) & $2 \mathrm{a}$ & $0 \mathrm{a}$ & $2 \mathrm{a}$ \\
\hline L4(E2/CC) & $0 \mathrm{a}$ & $0 \mathrm{a}$ & $1 \mathrm{a}$ \\
\hline \multicolumn{4}{|l|}{ Amostras } \\
\hline SF & $3 \mathrm{~A}$ & $0 \mathrm{~A}$ & $2 \mathrm{~A}$ \\
\hline CF & $0 \mathrm{~A}$ & $0 \mathrm{~A}$ & $0 \mathrm{~A}$ \\
\hline \multirow[t]{2}{*}{ CV (\%) } & 0.41 & 0.41 & 1.30 \\
\hline & \multicolumn{3}{|c|}{ 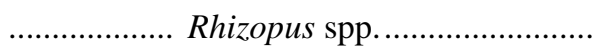 } \\
\hline \multicolumn{4}{|l|}{ Lotes } \\
\hline L1(E1/SC) & $0 \mathrm{a}$ & $1 \mathrm{a}$ & $0 \mathrm{a}$ \\
\hline L2(E1/CC) & $2 \mathrm{a}$ & $0 \mathrm{a}$ & $0 \mathrm{a}$ \\
\hline L3(E2/SC) & $0 \mathrm{a}$ & $0 \mathrm{a}$ & $0 \mathrm{a}$ \\
\hline L4(E2/CC) & $3 a$ & $0 \mathrm{a}$ & $0 \mathrm{a}$ \\
\hline \multicolumn{4}{|l|}{ Amostras } \\
\hline SF & $3 \mathrm{~A}$ & $0 \mathrm{~A}$ & $0 \mathrm{~A}$ \\
\hline CF & $0 \mathrm{~A}$ & $0 \mathrm{~A}$ & $0 \mathrm{~A}$ \\
\hline CV (\%) & 0.35 & 0.69 & 0.58 \\
\hline
\end{tabular}

Médias seguidas pela mesma letra (minúsculas para lotes e maiúsculas para amostras) não diferem entre si pelo teste Tukey, a 5\%.

ABRATES, Brasília, v.1, n.2, p.15-50, 1991.

MARCOS-FILHO, J. Teste de envelhecimento acelerado. In: VIEIRA, R.D. CARVALHO, N.M. (Ed.). Testes de vigor em sementes. Jaboticabal: FUNEP, 1994. p.133-149.

MARCOS-FILHO, J. Teste de envelhecimento acelerado. In: KRZYZANOWSKI, F.C.; VIEIRA, R.D.; FRANÇA-NETO, J. de 
B. (Ed.). Vigor de sementes, conceitos e testes. Londrina: ABRATES, 1999. 218p.

MARCOS-FILHO, J.; SHIOGA, P.S. Tratamento fungicida de sementes de soja no teste de envelhecimento rápido. Revista de Agricultura, Piracicaba, v.56, n.3, p.163-172, 1981.

MELLO, V.D.C.; TILLMANN, M.A.A. O teste de vigor em câmara de envelhecimento precoce. Revista Brasileira de Sementes, Brasília, v.9, n.2, p.93-102, 1987.

NASCIMENTO-JUNIOR, A.D.; TORRES, R.M.; FONSECA, E.P.; FARIA, A.L.R.; CARVALHO, N.M. Momento da realização do teste de germinação após o envelhecimento acelerado em sementes de soja. Revista Brasileira de Sementes, Brasília, v.20, n.1, p.223225, 1998.

PITT, J.L.; DYER, S.K.; McCAMMON, S. Systimic invasion of developing peanut plants by Aspergillus flavus. Letter Applied Microbiology, Oxford, v.13, n.1, p.16-20, 1991.

ROSETTO, C.A.V.; NAKAGAWA, J.; ROSOLEM, C.A. Efeito do meomento de colheita e da calagem na qualidade fisiológica de sementes de amendoim (Arachis hypogaea L.) cv. Botutatu. Revista Brasileira de Sementes, Brasília, v.16, n.2, p.138-146, 1994.

ROSSETTO, C.A.V.; BASSIN, C.A.; CARMO, M.G.F.; NAKAGAWA, J. Tratamento fungicida, incidência de fungos e momento de avaliação de germinação no teste de envelhecimento acelerado em sementes de amendoim. Revista Brasileira de Sementes, Londrina, v.23, n.2, p.78-87, 2001.

SANDERS, T.H.; HILL, R.A .; COLE, R.J.; BLANKENSHIP, P.D. Effect of drought on occurrence of Aspergillus flavus in maturing peanuts. Journal of the American Oil Chemists Society, Illinois, v.58, p. 966-970, 1981.

SCOTT, G.E. Improvement for accelerated aging response of seed in maize populations. Crop Science, Madison, v.21, n.1, p.41-43, 1981.
SILVA, M.A.D.; SILVA, W.R. Comportamento de fungos e de sementes de feijoeiro durante o teste de envelhecimento artificial. Pesquisa Agropecuária Brasileira, Brasília, v.35, n.3, p.599-608, 2000.

SINGH, K.; FRISVAD, J.C.; THRAME, U.L.F.; MATHUR, S.B. An illustrated manual on Identification of some Seed-borne Aspergilli, Fusara, Penicilia and their Mycotoxins. Copenhagen: Danish Government Institute of Seed Pathology for Developing Countries Ryvangs, 1992. 133p.

SMALL, H.; KVIEN, C.S.; SUMNERM M.E.; CSINOS, A.S. Solution Ca concentration and application date effect on pod calcium uptake and distribution in Florunner and Tifton-8 peanut. Journal of Plant Nutrition, NewYork, v.12, n.1, p.37-52, 1989.

SPINOLA, M.C.M.; CÍCERO, S.M.; MELO, M. Alterações bioquímicas e fisiológicas em sementes de milho causadas pelo envelhecimento acelerado. Scientia Agricola, Piracicaba, v.57, n.2. p.263-270, 2000.

TEKRONY, D.M.; EGLI, D.B.; BALLES, J.; TOMES, L.J.; STUCKEY, R.E. Effect of date of harvest maturity on soybean seed quality and Phomopsis spp. seed infection. Crop Science, Madison, v.24, n.1, p.189-193, 1984

USBERTI, R ; AMARAL, H.M. Fungicide dressing timing, seed size, seed origin and fungal incidence effects on groundnout (Arachis hypogaea L.) storability. Seed Science and Technology, Zürich, v.27, n.2, p.699-706, 1999

VANZOLINI, S.; NAKAGAWA, J. Teste de condutividade elétrica em genótipos de sementes de amendoim. Revista Brasileira de Sementes, Brasília, v.20, n.1, p.178-183, 1998.

ZANOTTO, M.D. Botutatu: nova cultivar de amendoim (Arachis hypogaea L.). Pesquisa Agropecuária Brasileira, Brasília, v.28, n.9, p. 1101-1102, 1993.

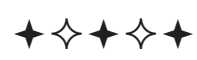

